occur at a non-transcribed gene. The findings indicate that Spt6 is needed to repress transcription initiation from a cryptic promoter in a gene by preserving the normal chromatin structure during pol II elongation.

So, taken together, one can envisage a model in which FACT facilitates pol-II-driven transcription by displacing H2A-H2B. The SPT16 subunit might also have histone chaperone activity, which helps reassemble the nucleosomes after transcription, and Spt6 and FACT might cooperate in this chromatin reassembly. It is not clear whether it is just the $\mathrm{H} 2 \mathrm{~A}-\mathrm{H} 2 \mathrm{~B}$ dimer that is displaced or, as the Winston data indicate, the entire nucleosome disassembles temporarily, so this remains to be determined in the future.

Arianne Heinrichs

(4) References and links ORIGINAL RESEARCH PAPERS

Belotserkovskaya, R. et al. FACT facilitates transcription-dependent nucleosome alteration. Science 301, 1090-1093 (2003) | Saunders, A. et al. Tracking FACT and the RNA polymerase II elongation complex through chromatin in vivo. Science 301, 1094-1096 (2003) | Kaplan, C. et al. Transcription elongation factors repress transcription initiation from cryptic sites. Science 301, 1096-1099 (2003)

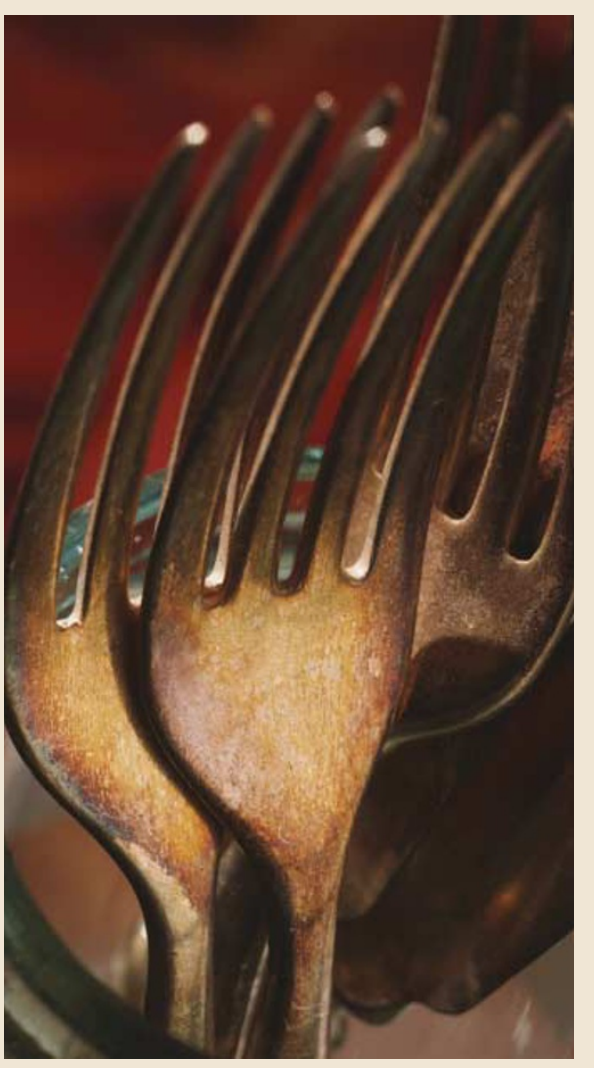

\section{Your own identity}

Although phosphatidylinositol-4-phosphate (PtdIns4P) is known to be an essential regulator of constitutive secretion from the late Golgi in yeast, its role in mammalian cells has remained unclear. It was thought to just be a substrate for the synthesis of phosphatidylinositol-4,5bisphosphate $\left(\operatorname{PtdIns}(4,5) \mathrm{P}_{2}\right)$, a known essential regulator of plasma-membrane trafficking. However, the Golgi contains only low levels of $\operatorname{PtdIns}(4,5) \mathrm{P}_{2}$ and no direct role for $\operatorname{PtdIns}(4,5) \mathrm{P}_{2}$ in Golgi-membrane trafficking has been established. So, could PtdIns4P itself have a more important role in the Golgi? In Cell, Yin and colleagues now show that the answer to this question is yes.

The authors first established that the phosphatidylinositol 4-kinase (PI4K) PI4KII $\alpha$ - which makes PtdIns4P - is a Golgi resident enzyme, and then used small interfering RNA (siRNA) to study the role of this enzyme in the mammalian Golgi. They showed that PI4KII $\alpha$ siRNA significantly reduced the incorporation of ${ }^{32} \mathrm{P}$ into both PtdIns4P and $\operatorname{PtdIns}(4,5) \mathrm{P}_{2}$. Furthermore, they used immunofluorescence to show that PtdIns4P is most concentrated in the Golgi (surprisingly little PtdIns4P was present at the plasma membrane), and that PI4KII $\alpha$ siRNA selectively decreased these Golgi PtdIns4P levels.

Next, Yin and co-workers showed that PI4KII $\alpha$ siRNA markedly reduced the association of $\gamma$-adaptin - a subunit of the adaptor protein (AP)-1 complex - with the Golgi. AP-1 regulates clathrin-coated-vesicle trafficking between the trans-Golgi network and the endosome/lysosome system, so, as expected, the authors found that the levels of Golgi clathrin were also significantly reduced. By contrast, $\beta$-COP - a subunit of the coatomer protein (COP)I complex — remained associated with the Golgi.

The decreased association of $\gamma$-adaptin with the Golgi after the addition of PI4KII $\alpha$ siRNA could be due to a lack of PtdIns4P or PtdIns $(4,5) \mathrm{P}_{2}$, so the authors used a solid-phase, lipid-binding assay to determine which lipid regulates the recruitment of AP- 1 to the Golgi. They found that AP-1 binds PtdIns4P, but not PtdIns $(4,5) \mathrm{P}_{2}$, so PtdIns4P is probably the main recruiter of AP-1. Interestingly, this means that the lipid specificities of AP- 1 and AP-2 are different - AP-2 preferentially binds PtdIns $(4,5) \mathrm{P}_{2}$ in the plasma membrane.

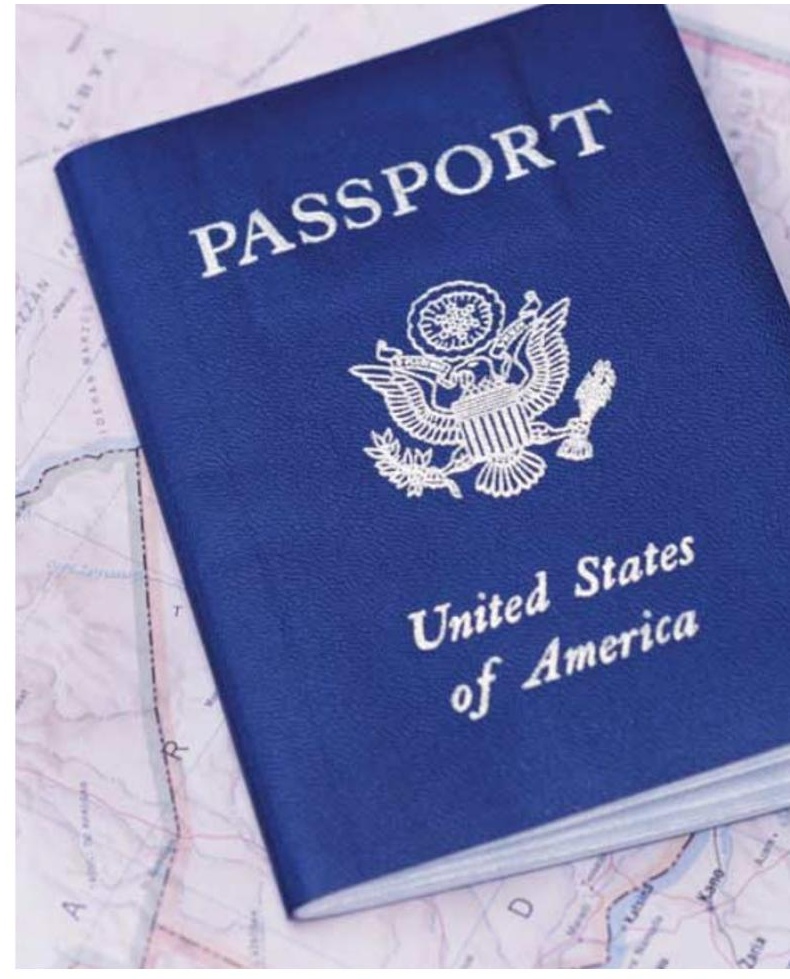

To verify that PtdIns4P is the main recruiter of AP-1 to the Golgi in vivo, the authors used three methods. First, they shuttled PtdIns4P or PtdIns $(4,5) \mathrm{P}_{2}$ into live cells using membranepermeant polyamine carriers after the addition of PI4KII $\alpha$ siRNA. They found that shuttling PtdIns4P into cells rescued AP-1 recruitment, whereas shuttling PtdIns $(4,5) \mathrm{P}_{2}$ did not. They saw the same effect using their second method, in which exogenous PtdIns4P or PtdIns $(4,5) \mathrm{P}_{2}$ was added back to semi-intact cells after PI4KII $\alpha$ siRNA. In the third method, they showed that anti-PtdIns4P antibodies selectively inhibited AP1 Golgi recruitment. These data show that PtdIns4P is a crucial Golgi lipid that functions independently of PtdIns $(4,5) \mathrm{P}_{2}$.

On the basis of this work, Yin and colleagues propose that “...PI4KII $\alpha$ establishes the Golgi's unique lipid-defined organelle identity by generating $[\mathrm{PtdIns4} \mathrm{P}]$-rich domains that specify the docking of the AP-1 coat machinery". In addition, they propose that, as the plasma membrane is enriched in $\operatorname{PtdIns}(4,5) \mathrm{P}_{2}$ and endosomes are enriched in phosphatidylinositol3-phosphate, Golgi PtdIns4P might represent "...the third arm of the membrane lipid recognition system".

Rachel Smallridge

\section{(0) References and links}

ORIGINAL RESEARCH PAPER Wang, Y. J. et al. Phosphatidylinosito 4 phosphate regulates targeting of clathrin adaptor AP-1 complexes to the Golgi. Cell 114, 299-310 (2003)

FURTHER READING Bonifacino, J. S. \& Lippincott-Schwartz, J. Coat proteins: shaping membrane transport. Nature Rev. Mol. Cell Biol. 4, 409-414 (2003)

WEB SITE

Helen Yin's laboratory: http://www3.utsouthwestern.edu/yinlab 